\title{
Magnetotelluric analysis using Mohr circles
}

\author{
F. E. M. Lilley*
}

\section{ABSTRACT}

The Mohr circle, most commonly met in the analysis of mechanical stress, is used to depict magnetotelluric impedance information, taking the real and quadrature parts of magnetotelluric tensors separately. The magnetotelluric concepts of two-dimensionality, three-dimensionality, skew and anisotropy are then all given quantitative expression on a diagram, as are various magnetotelluric invariants. In particular, a new invariant, the "central impedance," becomes evident in a discussion of effective impedances. Some insight is gained into impedance rotations, and an anisotropy angle is defined, analogous to skew angle.

Mohr circles are also tested to depict the effects of the shear and twist operations on a regionally twodimensional structure. Generally, the application of shear or twist results in an impedance tensor with a Mohr circle of typical three-dimensional form.

\section{INTRODUCTION}

Mohr circles are known in geophysics as a way to relate shear stress and normal stress in a body that is subjected to mechanical pressure. As Jaeger (1969) notes, “. . . Mohr's diagram provides the most elegant representation of the variation of normal and shear stress with direction ....".

Mohr circles thus depict stress tensors and are most common in the basic case of stress in two dimensions; however, they are also used for stress in three dimensions, and to depict strain (Jaeger and Cook, 1976).

Originally used in the graphical solution of a variety of stress and strain problems, Mohr circles achieve the objective of plotting, uniquely on a simple diagram, the four different values that comprise a $2 \times 2$ tensor. This achievement results from taking two degrees of freedom to plot a Cartesian point for the center of the circle, and then two more degrees of freedom to give the radius of the circle and the bearing of its commencement point. Thus, four degrees of freedom are taken in the combination of a polar plot with a Cartesian plot.

In addition to stress and strain, Mohr circles are of general application (Nye, 1957) in representing tensor information by diagrams, and were introduced for magnetotelluric data in an earlier paper (Lilley, 1976). In magnetotellurics, as in much of geophysics, it is of central importance to know whether interpretation may proceed in terms of simplified structures; that is, to know whether a one-dimensional, two-dimensional, or three-dimensional (1-D, 2-D, or 3-D) geologic model is appropriate. The last decade has seen a development of interest in methods for analysing a magnetotelluric impedance tensor for this sort of information. General analysis has been advanced in a series of papers by Eggers (1982), Spitz (1985), LaTorraca et al. (1986), and Yee and Paulson (1987). Another line of inquiry has been the development of particular models, involving a combination of local and regional effects. Amongst recent contributions to this topic are those of Park (1985), Counil et al. (1986), Zhang et al. (1987), Ingham (1988) Park and Livelybrooks (1989), Bahr (1988, 1991) and Groom and Bailey (1989, 1991).

As shown in Lilley (1976), Mohr circles can be used not only to represent magnetotelluric tensors for 2-D conductivity structures with the symmetry traditionally associated with Mohr circles, but also to represent magnetotelluric tensors for 3-D conductivity structures (still observed in two dimensions of measurement on the surface of the earth). These latter tensors lose the traditional symmetry, and their circles -exhibit an angular offset that may be regarded as a skew angle. Thus 1-D data plot as a single point, 2-D data as a circle centered on the horizontal axis, and 3-D data as a circle centered off the horizontal axis.

In the present paper, the application of the Mohr circle method to the display of magnetotelluric data is further explored. The concept of rotation of an observed tensor, corresponding to calculating the values that would be observed were the measuring axes to be physically rotated, is central to the method.

Also, real and quadrature parts of a tensor are dealt with separately. This procedure is a departure from much common practice, but may offer a useful alternative. The ulti-

Manuscript received by the Editor October 23, 1991; revised manuscript received March 3, 1993.

* Research School of Earth Sciences, Australian National University, GPO Box 4, Canberra A.C.T. 2601, Australia.

(C) 1993 Society of Exploration Geophysicists. All rights reserved. 
mate objective of such magnetotelluric tensor rotation is to enable judgments to be made concerning questions such as the following: How well does the observed situation approximate the ideal of two-dimensionality? What, then, is the best direction to take as 2-D strike? What are the best values to take for the along-strike and across-strike impedances? The present paper advances the view that displaying magnetotelluric data in Mohr circles as real and quadrature parts separately may help in these judgments.

The method is explored by seeking directions for the Eggers (1982) and LaTorraca et al. (1986) analyses, when the real and quadrature parts of a tensor are examined separately. Another traditionally important concept in magnetotelluric studies has been that of invariance under the rotation of axes, and a number of traditional magnetotelluric invariants are discussed, again taking the real and quadrature parts of a tensor separately. While focusing on the invariants of Berdichevsky and Dmitriev (1976) the existence of a third invariant becomes evident, which is basic in terms of Mohr circle representation. This new invariant is termed the "central impedance."

The angle characterising the three-dimensionality of a Mohr circle diagram corresponds to a skew angle. Similarly, another angle is evident that characterizes anisotropy, and is advanced as the "anisotropy angle."

Finally, the method is applied to examples of the Groom and Bailey (1989) situation, where local twist and shear operate on a tensor that is regionally 2-D. Certain consequences of twist and shear are depicted and recognized.

Modern computational procedures have changed the status of Mohr circles. While their earlier use in the graphical solution of computational problems is lessened, they may now be produced immediately by modern graphics, with color to show variation with frequency (in the case of magnetotellurics). Their convenience and usefulness as a diagnostic tool is thus enhanced.

\section{THE MOHR CIRCLES}

Circle for data from a 2-D structure

The notation of Groom and Bailey (1989) is adopted in this paper for a magnetotelluric tensor which is 2-D in form. When rotated so that the axes are parallel and perpendicular to strike, the tensor is denoted

$$
\left[\begin{array}{ll}
Z^{\prime} x x & Z^{\prime} x y \\
Z^{\prime} y x & Z^{\prime} y y
\end{array}\right]=\left[\begin{array}{cc}
0 & a \\
-b & 0
\end{array}\right],
$$

with

$$
\sigma=a+b
$$

and

$$
\delta=a-b
$$

Then one-dimensionality of electrical conductivity structure, for which

$$
a=b \text {, }
$$

is given by

$$
\delta=0
$$

and extreme anisotropy, for which is given by

$$
|a| \gg|b|
$$

$$
\delta \simeq \sigma .
$$

Additional notations in this paper are subscripts $r$ and $q$ for the real and quadrature parts of an entity.

The Mohr circles are generated when the real and quadrature parts of a tensor are considered separately under the rotation of axes. A plot of $Z^{\prime} x x$ against $Z^{\prime} x y$, as the measuring axes rotate, then describes a circle, where $Z^{\prime} x x$ and $Z$ 'xy are the values that would be measured with axes rotated angle $\boldsymbol{\theta}^{\prime}$ clockwise from those that gave the initial values of Zxx and Zxy . As shown in Figure la, such a circle for the real parts of the tensor elements is centered on the Z'xyr-axis at point $\left(\sigma_{r} / 2,0\right)$ and is of radius $\delta_{r} / 2$.

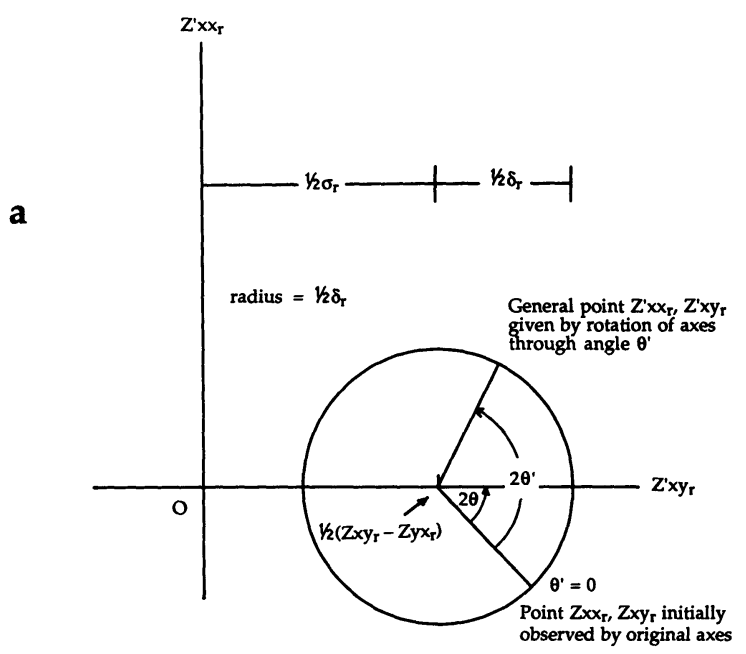

AXIS ROTATION
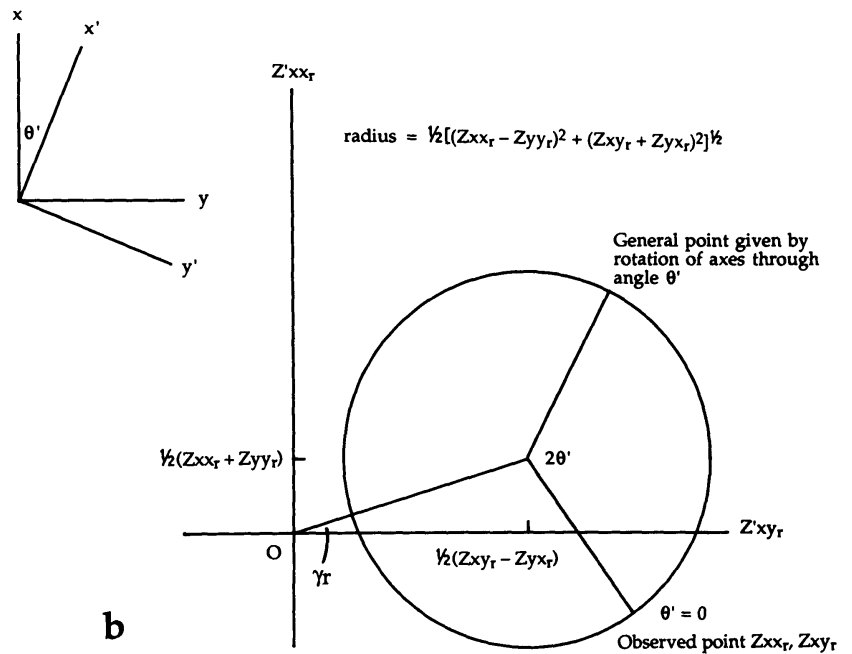

FIG. 1. Graphic representation of the values taken by the real elements of a magnetotelluric impedance tensor upon rotation of the measuring axes through angle 8'; (a) for induction in 2-D structure, and (b) for induction in general 3-D structure. Similar diagrams can be drawn for the quadrature elements. Note that the notations $Z_{A}$ and $Z_{B}$ of Lilley (1976) here become $\delta_{r} / 2$ and $\sigma_{r} / 2$, and that $\tan \gamma_{r}$ is a skew value for the real part of the tensor by itself. For 1-D structure, the circle in (a) reduces to its central point. 
For a 1-D case,

$$
b=a,
$$

and

$$
\delta=0
$$

and the circle reduces to its central point.

The angle $\theta$ (clockwise) necessary to rotate the measuring axes for alignment with the principal axes (to give the two points where the circle intercepts the $Z^{\prime} x y_{r}$-axis) is also part of Figure la, entering as a rotation of the radial arm by angle 28 (anticlockwise) from its initial observed position at

$$
\theta^{\prime}=0 .
$$

A similar circle will also exist for the quadrature parts of $a$ and $b$, with center $\left(\sigma_{q} / 2,0\right)$ and radius $\delta_{q} / 2$. The real and quadrature circles will be distinct from each other; however, for a true 2-D case they must show the same angle 28 for rotation to 2-D alignment.

Circles for data from a 3-D structure

For a general case of a tensor indicating 3-D electrical conductivity structure, the circle is offset from the Z'xyaxis, as shown in Figure lb. The offset can be expressed by an angle $\gamma_{r}$ as shown. The value of $\tan \gamma_{r}$ is of a form similar to the "skew" of Swift (1967), and the offset angle $\gamma_{r}$ shows the skew of the real part of the tensor graphically.

\section{CONNECTION WITH THE EGGERS ANALYSIS}

Eggers (1982) considered rotation of the impedance tensor and (using an eigenstate formulation) sought two polariza-

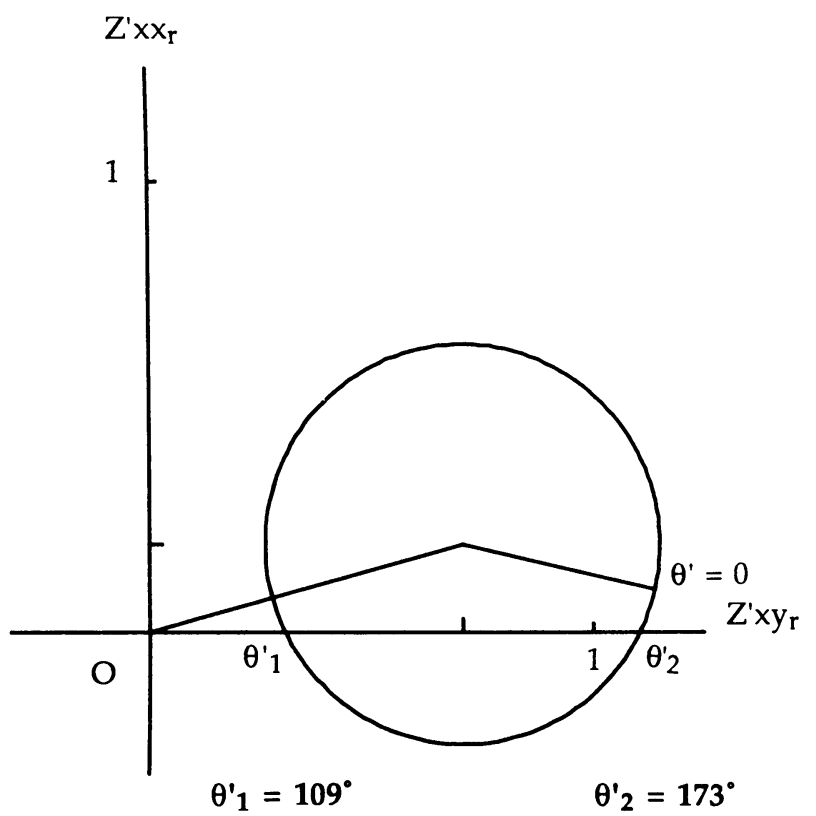

tion states, not necessarily orthogonal to each other, for which the electric and magnetic fields have the same polarization at perpendicular orientations (see also the treatment of this problem by Yee and Paulson, 1987). When real and quadrature parts of an impedance tensor are considered separately, the ellipses sought by Eggers reduce to linear polarizations, and their azimuths can be seen by inspection of the appropriate Mohr circles.

The tensor quoted by Eggers (1982) is thus shown in Figure 2, with real and quadrature circles. What may now be sought, for each circle, are two directions for which the electric and magnetic fields are at perpendicular orientations.

Taking the real circle first, these directions are clearly those corresponding to the values of $\theta^{\prime}$ for which the circle cuts the $Z^{\prime} x y_{r}$-axis; for it is at these points that $Z^{\prime} x x_{r}$ is zero, and so an applied magnetic field $\boldsymbol{H}^{\prime} x$ will produce an electric field E'y orthogonal to it. The actual values of $\theta^{\prime}$ are given in the figure. Two other values of $\theta^{\prime}$, also given in the figure, are similarly found for the quadrature circle.

\section{CONNECTION WITH THE LATORRACA ET AL. ANALYSIS}

LaTorraca et al. (1986) considered rotation of a complex impedance tensor in a different way, that is to obtain two mutually orthogonal magnetic field directions for which the associated electric fields are orthogonal to each other (but not necessarily orthogonal to the magnetic fields).

Considering real and quadrature parts separately, and the real part first, the LaTorraca directions are shown most directly on a plot of $Z^{\prime} x x_{r}$ against $Z^{\prime} y x_{r}$, as in Figure 3. Then, two points of fixed $Z^{\prime} x x_{r} / Z^{\prime} y x_{r}$ ratio [the LaTorraca et al. (1986) condition for the direction of $E$ fields due to an applied $\boldsymbol{H}^{\prime} \boldsymbol{x}$ ] are given by the intersections with the circle of

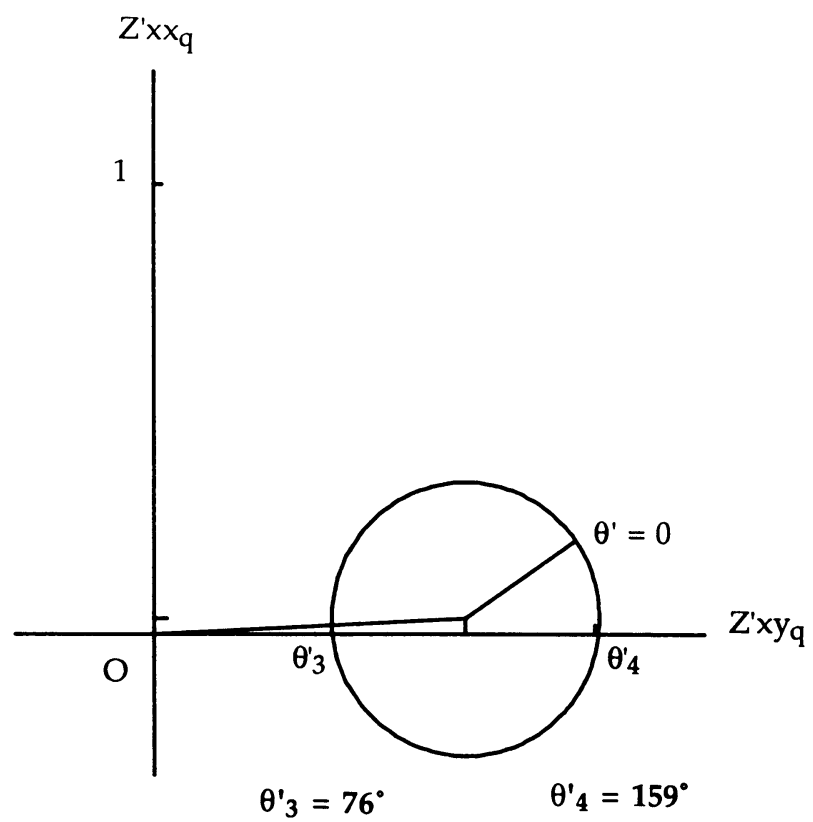

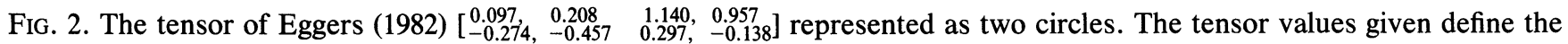

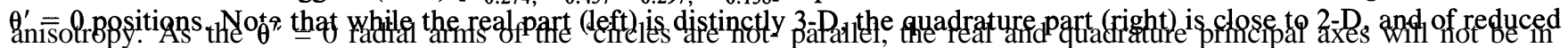
alignment. The intercepts of the circles with the $Z^{\prime} x y,-$ and $Z^{\prime} x y, q-a x e s$, at $\theta_{1}^{\prime}, \theta_{2}^{\prime}, \theta_{3}^{\prime}$, and $\theta_{4}^{\prime}$ as marked, indicate the appropriate rotations (values of $\theta^{\prime}$ ) for the Eggers polarization states, taking real and quadrature parts separately. 
a line from the origin to the circle's center, continued to cut the circle a second time. Such constructions are shown in Figure 3 for both the real and quadrature parts of the Eggers tensor example, and the values of the rotation angle $\theta^{\prime}$ thus found are given. These values of $\boldsymbol{\theta}^{\prime}$ can be compared with the values determined in Figure 2 for the Eggers directions; the discrepancies are a consequence of the 3-D nature of the data.

Clearly, for a 2-D tensor, the Eggers and LaToracca et al. directions agree and become the directions of the 2-D principal axes.

\section{INVARIANTS UNDER ROTATION OF THE MEASURING AXES}

Berdichevsky and Dmitriev (1976) identified two impedances that are invariant under rotation of the measuring axes. These impedances, which are complex, are termed "effective impedances" and are

$$
Z_{\text {eff }}^{I}=(Z x x Z y y-Z x y Z y x)^{1 / 2},
$$

which is commonly called the determinantal impedance, and

$$
Z_{e f f}^{I I}=(Z x y-Z y x) / 2,
$$

which is sometimes known as the "average" or "trace" impedance. Applying these expressions to the real and quadrature parts of a tensor separately (as distinct from taking the real and quadrature parts of $\boldsymbol{Z}_{\text {eff }}^{I}$ and $\boldsymbol{Z}_{\text {eff }}^{I I}$ ) produces quantities that are also invariant, and which may be identified on the Mohr circle depiction of an impedance tensor, as will now be shown.

The real part of the Eggers tensor is shown again as an example in Figure 4, where now axes are included for all of $Z^{\prime} x x_{r}, Z^{\prime} x y, Z^{\prime} y x_{r}$, and Z'yy, following the symmetry relations indicated in Lilley (1976). Thus, for any point on the circle (corresponding to some particular axis rotation), values for all the tensor elements can be scaled off. In this case $Z^{\prime} x y_{r}$ is always positive, $Z^{\prime} y x_{r}$ always negative, and $Z^{\prime} x x_{r}$ and $Z^{\prime} y y$, range both positive and negative. The circle has radius $R Q$, and distances $d_{1}, d_{2}, d_{3}$, and $d_{4}$ are as drawn on the fogure.

With reference to Figure 4, by the theorem of Pythagorus it is evident that

$$
d_{3}^{2}=R Q^{2}+d_{2}^{2}
$$

and

$$
d_{3}^{2}=d_{1}^{2}+d_{4}^{2}
$$

so that

$$
d_{2}^{2}=d_{1}^{2}+d_{4}^{2}-R Q^{2} .
$$

Because, from Figure $1 b$,

$$
\begin{aligned}
& d_{1}=\left(\mathrm{Zxy},-Z y x_{r}\right) / 2 \\
& d_{4}=\left(Z x x_{r}+Z y y_{r}\right) / 2
\end{aligned}
$$

and

$$
R Q=\frac{1}{2}\left[\left(Z x x_{r}-Z y y_{r}\right)^{2}+\left(Z x y_{r}+Z y x_{r}\right)^{2}\right]^{1 / 2}
$$

it follows that

$$
d_{2}=\left(Z x x_{r} Z y y_{r}-Z x y_{r} Z y x_{r}\right)^{1 / 2} .
$$

Thus, the distance $d_{2}$ on Figure 4 , an invariant for the circle, can be seen to represent the "first effective" or determinantal impedance, when only real parts of the tensor elements are considered. Similarly the distance $d_{1}$ on Figure 4 represents the "second effective" impedance, taking real parts of the tensor elements only. (In this case, because of the simplicity of the expression, $\boldsymbol{d}_{1}$ is also the real part of the full complex " "second effective' 'impedance.) As can be seen on the figure, $d_{1}$ is greater or less than $\boldsymbol{d}_{2}$ depending on whether
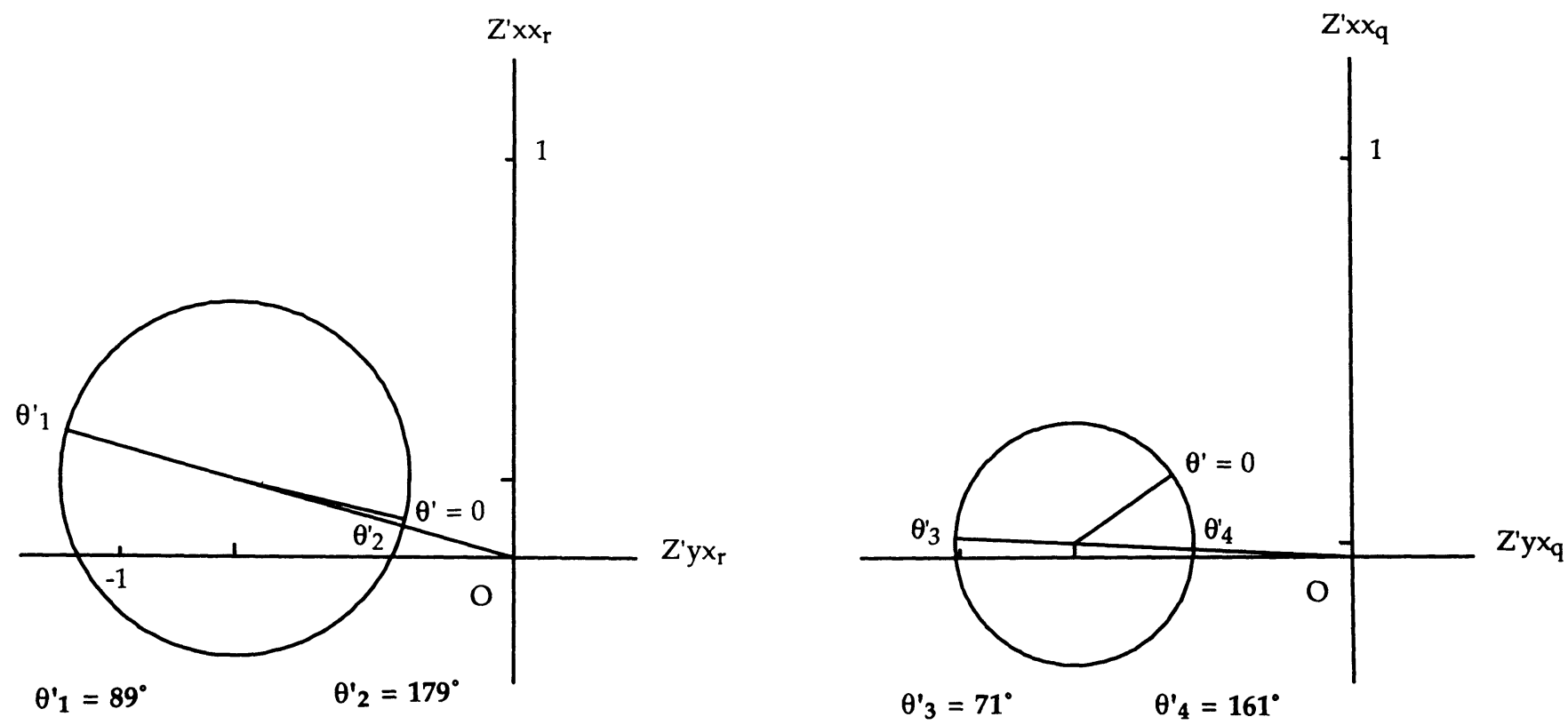

FIG. 3. The Eggers tensor plotted as circles of Z'xx versus Z'yx, with constructions shown to give the angles of rotation for the magnetic field directions of LaTorraca et al. (1986). These angles are $\boldsymbol{\theta}_{1}^{\prime}$ and $\boldsymbol{\theta}_{2}^{\prime}$ for the real part of the tensor (left), and $\boldsymbol{\theta}_{3}^{\prime}$ and $\boldsymbol{\theta}_{\mathbf{4}}^{\prime}$ for the quadrature part (right). 
the radius of the circle is greater or less than $\mathrm{d}_{4}$ (which is in some respects a balance of anisotropy against skew).

A diagram similar to Figure 4 may also be drawn for the quadrature elements of a tensor, with its own invariant distances that correspond to $d_{1}$ and $\mathrm{d}_{2}$ on Figure 4 .

A new invariant, the central impedance

Inspection of Figure 4 suggests that the distance from the origin to the center of the circle, line OQ, is another significant invariant. In terms of the Mohr circle representation, the center of the circle is the most central invariant point of the tensor figure. Thus the length $d_{3}$ of the line OQ, given by

$$
d_{3}=\frac{1}{2}\left[\left(Z x x_{r}+Z y y_{r}\right)^{2}+\left(Z x y_{r}-Z y x_{r}\right)^{2}\right]^{1 / 2}
$$

is a basic invariant impedance, and is termed here the "central impedance." There is also a central impedance value for the quadrature part of the tensor, with subscripts $q$ replacing subscripts $r$ in the expression just given.

An anisotropy angle

Further, in Figure 4 the circle radius RQ is a natural measure of the tensor anisotropy, perhaps best normalized by the distance $d_{2}$. Thus an anisotropy index $p_{r}$ for the tensor is evident as

$$
\begin{aligned}
p_{r} & =R Q / d_{2} \\
& ={ }^{1}\left[\left(Z x x_{r}-Z y y_{r}\right)^{2}+\left(Z x y_{r}\right.\right. \\
& \left.\left.+Z y x_{r}\right)^{2}\right]^{1 / 2} /\left(Z x x_{r} Z y y_{r}-Z x y_{r} Z y x_{r}\right)^{1 / 2} .
\end{aligned}
$$

Index $p_{r}$ thus ranges in value from zero for a 1-D case (consider the circle in Figure la reduced to its central point) to infinity (the consequence, as explained in the introduction above) of

$$
\delta \rightarrow \sigma
$$

Analogous to skew angle $\gamma_{r}$ having a tangent of $d_{4} / d_{1}$ in Figure 4, angle $R O Q$ is noted to be an "anisotropy angle," $\lambda_{r}$, equal to $\arctan p_{r}$. That is,

$$
\lambda_{r}=\arctan p_{r} .
$$

A similar index $p_{q}$ and angle $\lambda_{q}$ exist for the quadrature part of a tensor.

For the Eggers (1982) tensor of Figure 2, the values of the anisotropy indices and angles are:

$$
\begin{array}{ll}
p_{r}=0.76, & \lambda_{r}=37^{\prime} \\
p_{q}=0.48, & \lambda_{q}=25^{\circ}
\end{array}
$$

with skew angles being

$$
\gamma_{r}=16^{\circ} \quad \text { and } \quad \gamma_{q}=3^{\prime} .
$$

Note that for this same complex tensor Eggers (1982) obtained a skew value of 12 degrees and LaTorraca et al. (1986) a skew value of 11 degrees. These values fall within the range of $\gamma_{r}$ and $\gamma_{q}$ determined above.

\section{FREQUENCY DEPENDENCE}

An important aspect of observed magnetotelluric data is their frequency dependence. The Mohr circle method described so far is for data at a particular frequency, (or averaged over some frequency band). Data over a full range of frequencies may be displayed by color graphics using a color spectrum to cover the frequency range. This method is displayed here in principle, in Figure 5.

Figure 5 shows as an example, magnetotelluric data over a range of four frequencies. The data are from a seafloor site (TP3) in the Tasman Sea recorded by the instruments of Filloux (1987) and reduced by Ferguson (1988). The experiment is described in Lilley et al. (1989). Just four frequencies are shown for clarity; the values of the observed tensor elements have been multiplied by a factor of $\sqrt{T}$ in each case (where $T$ is the period in hours) to normalize them against

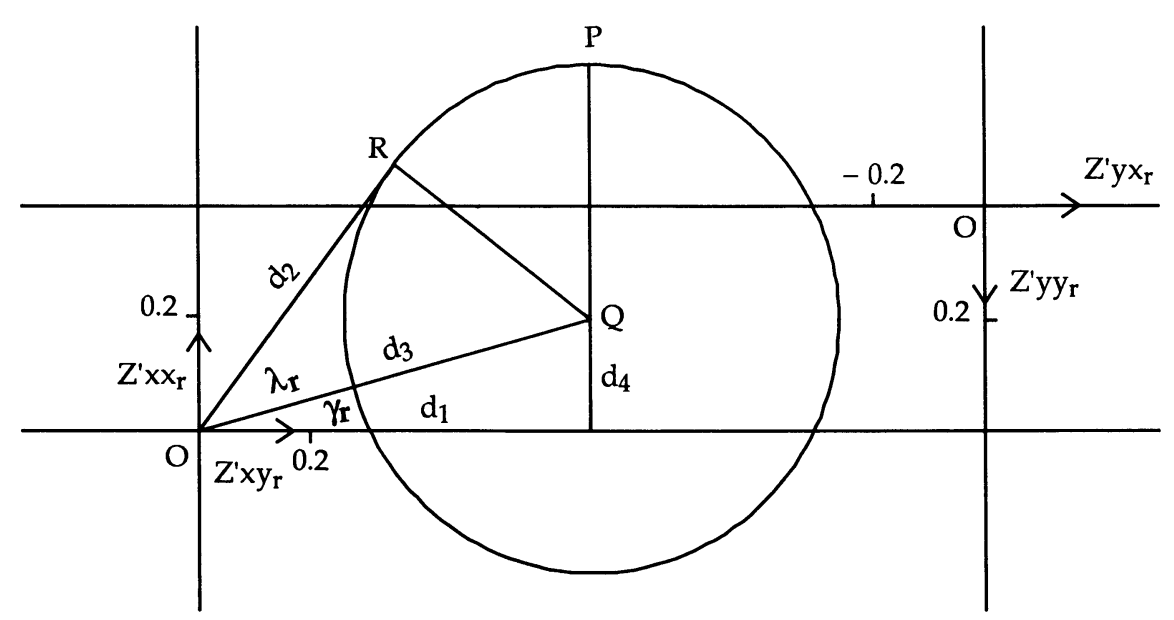

FIG. 4. The real part of the Eggers tensor presented as a circle, with axes for all four tensor elements included. The figure shows the construction to give $\mathrm{d}_{2}$, the magnitude of the determinantal impedance (considering only real parts). The second effective (or "trace") impedance is of magnitude $\mathrm{d}_{1}$. The "central impedance," now proposed as a basic invariant is $\mathrm{d}_{3}$, the line joining the origin to the center of the circle. Angle $\gamma_{r}$ is the skew angle, and angle $\lambda_{r}$ the "anisotropy angle." 
the characteristic that, above a uniform half-space, such tensor elements are proportional to $T^{-1 / 2}$.

The circles show clearly that the observed data are 2-D in character and highly anisotropic. (These points are important for marine data, as discussed by Ferguson et al. 1985, 1990, and Heinson, 1991.) Phase information is also evident in the relative sizes of the real and quadrature circles changing with frequency.

\section{APPLICATION TO GROOM-BAILEY DECOMPOSITION}

Groom and Bailey (1989) consider the case where a tensor that is regionally 2-D as in equation (1) is distorted locally by shear, twist, and anisotropy. Introducing notation

$$
\begin{aligned}
& \alpha_{0}=Z x x+z y y \\
& \alpha_{1}=Z x y+Z y x \\
& \alpha_{2}=Z y x-Z x y \\
& \alpha_{3}=Z x x-z y y
\end{aligned}
$$

they show that these processes affect a measured magnetotelluric impedance tensor

$$
\left[\begin{array}{ll}
Z x x & Z x y \\
Z y x & Z y y_{1}
\end{array}\right.
$$

such that

$$
\begin{aligned}
& \alpha_{0}=\text { to }+e \delta \\
& \alpha_{1}=(\delta-\text { eta }) \cos 2 \theta-(t \delta+\text { ea }) \sin 28 \\
& \alpha_{3}=-\sigma+\text { et } 8 \\
& \alpha_{3}=-(t \delta+e o) \cos 28-(6-\text { eta }) \sin 2 \theta,
\end{aligned}
$$

where

1) the measurement axes are rotated angle $\theta$ from the regional 2-D axes $\left(0^{\circ} \leq \theta \leq 90^{\circ}\right)$,

2) parameter $e$ is the shear parameter which affects the regional 2-D tensor as multiplication by a factor of $\left(1+e^{2}\right)^{-1 / 2}\left[\begin{array}{cc}1 & e \\ e & 1\end{array}\right]$, and
3) element $t$ is the twist parameter which summarizes the twist effect on the regional tensor by multiplying it by

$$
\left(1+t^{2}\right)^{-1}\left[\begin{array}{ccc}
2 & 1 & -t \\
t & 1 & 1
\end{array}\right] .
$$

Given observed values of $Z x x, Z x y, Z y x$, and Zyy, in the Groom-Bailey scheme the real and quadrature equations (2) are solved for $t, \mathrm{e}, \boldsymbol{\theta}, \boldsymbol{\delta}$, and a; i.e., eight equations for seven unknowns (as $\delta$ and $\sigma$ are complex).

In this paper now note that equations (2) may be expressed

$$
\begin{aligned}
\alpha_{0}= & \sigma(\tan A+\tan C) \\
\alpha_{1}= & \delta\{(1-\tan A \tan B) \cos 28 \\
& -(\tan A+\tan B) \sin 2 \theta\} \\
\alpha_{2}= & -\sigma(1-\tan A \tan C) \\
\mathrm{a} 3= & \delta\{-(\tan A+\tan B) \cos 2 \theta \\
& -(1-\tan A \tan B) \sin 2 \theta\},
\end{aligned}
$$

where $A$ denotes the Groom-Bailey "twist" angle, so that

$$
\tan A=\mathrm{t}
$$

and the further notation has been introduced of

$$
\tan B=\eta=e \sigma / \delta
$$

and

$$
\tan \mathrm{C}=\zeta=e \delta / \sigma .
$$

In Figure 6 below, notations are also used of

$$
\tan D=\delta / \sigma
$$

and

$$
\tan E=\text { e. }
$$

Here it is generally understood that analysis proceeds separately for the real and quadrature parts, with appropriate subscripts understood: thus, for example, $\eta_{r}=e \sigma_{r} / \delta_{r}$ and $\tan B_{r}=\eta_{r}$ (note $\eta_{r}$ is not the real part of $e \sigma / \delta$, where $\sigma$ and $\delta$ are complex).
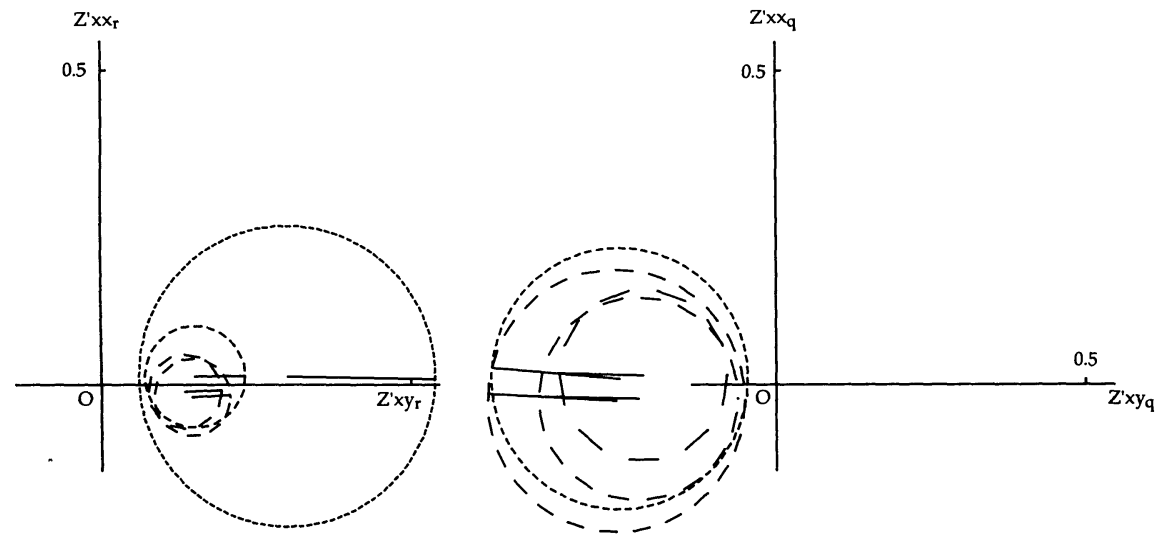

FIG. 5. Magnetotelluric impedance data from a seafloor site in the Tasman Sea from Ferguson (1988) taken as an example for plotting over a frequency range. The periods are $0.19 .0 .48,1.44$, and $4.44 \mathrm{hr}$, and increasing period correspends to increasing length of dashed line. Before plotting, the impedance values have been normalized by multiplication by $\sqrt{T}$. 
a Shear only
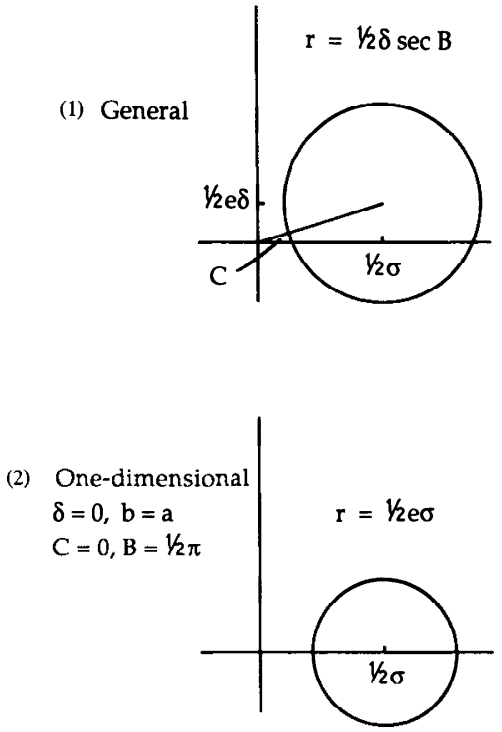

(3)
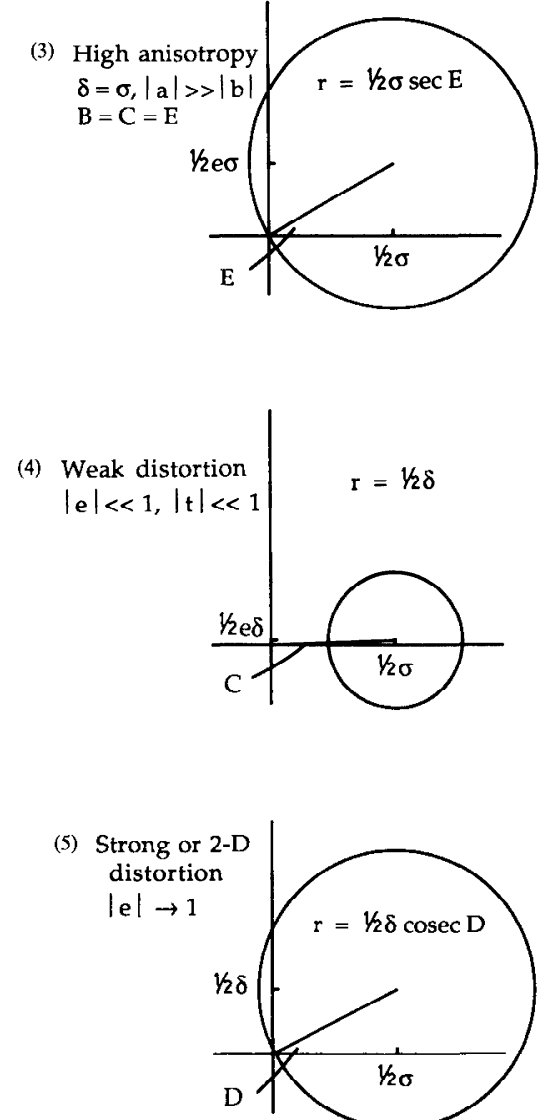

b Twist only
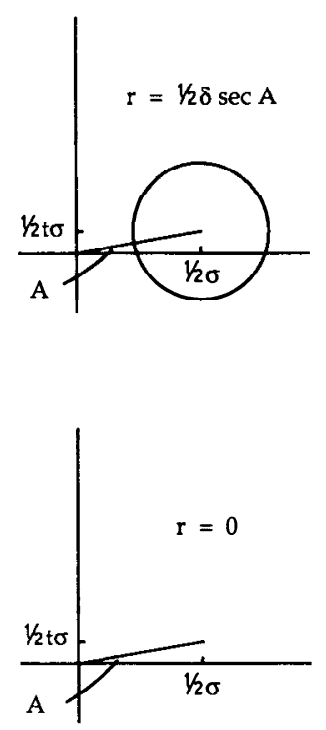

c Both shear and twist
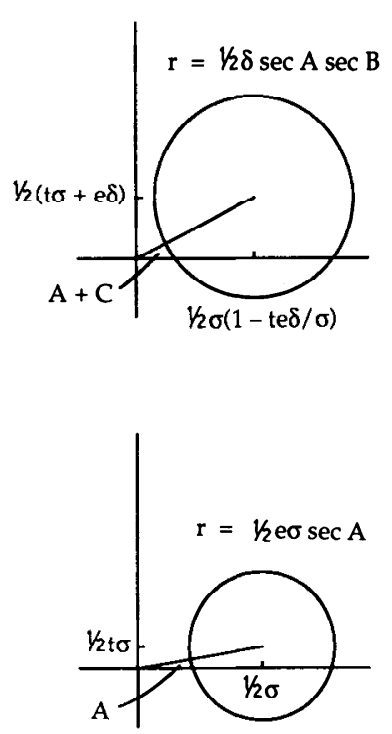
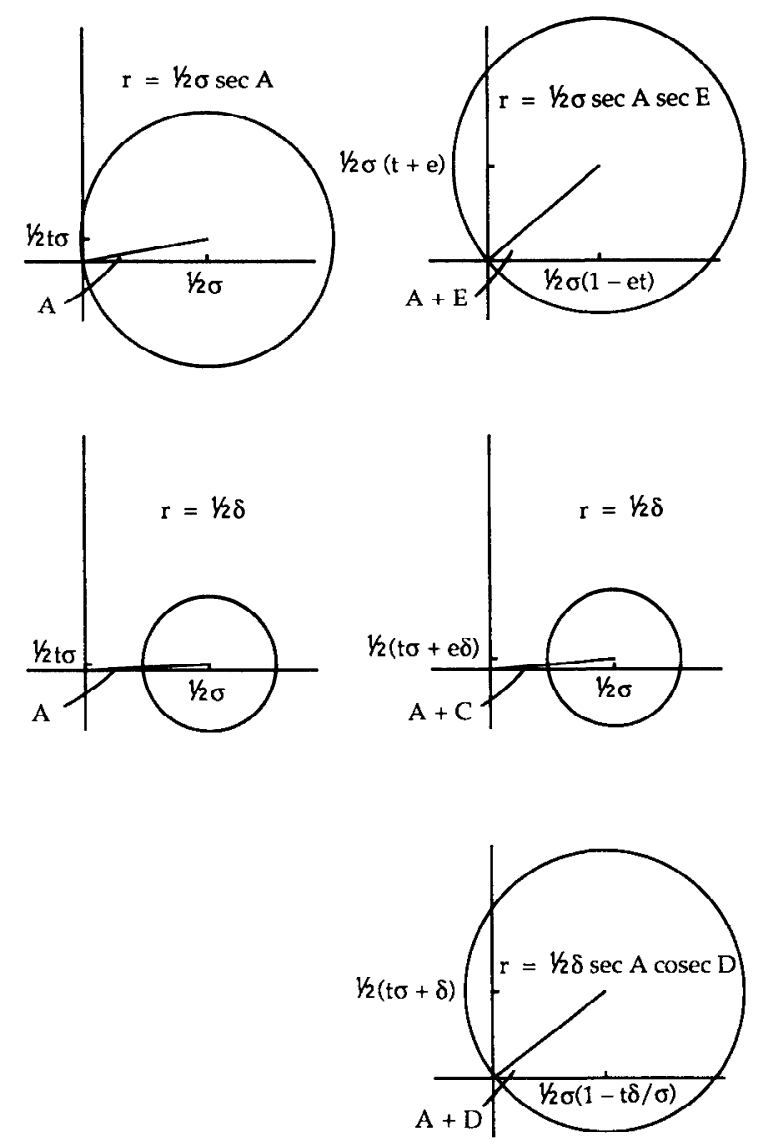

FIG. 6. Set of diagrams, of the form of Figure $1 b$, for both general and particular cases of a Groom-Bailey magnetotelluric impedance tensor. a) Shear only; b) Twist only; c) Both shear and twist. Except where stated otherwise, the examples have been drawn taking $6=0.23, \sigma=0.43$, e $=0.58$, and $t=0.18$. Each axis in the figure covers the range -0.1 units to +0.4 units. In this figure, $r$ denotes circle radius. 
Some algebra then shows that

$$
\tan (A+C)=-\alpha_{0} / \alpha_{2}
$$

so that, when defining skew angles for the real and quadrature parts of a tensor separately, these will be given by $(A+$ $\mathrm{C}$,$) and \left(A+C_{q}\right)$.

It also follows from the above equations that

$$
\tan (A+B+28)=-\alpha_{3} / \alpha_{1} .
$$

Depicting the decomposition graphically

The relationships of equations (2) and (3) can be directly expressed by the diagrams in Figure 1. A suite of such diagrams is presented in Figure 6, for both general and particular cases. It is instructive if shear and twist are introduced separately, and then combined, and in Figure 6 this procedure is adopted in columns (a), (b), and (c), respectively.

There are five rows in Figure 6: row (1) for general cases, and rows (2-5) for particular cases considered by Groom and Bailey (1989) which may have distinctive characteristics.

Some of the main features of Figure 6 are now discussed.

1) Shear but no twist $(e \neq 0, t=0)$; Figure 6 , column (a).-The general case for shear only is shown in Figure $6 a$ row (1). Note that, relative to the undistorted 2-D tensor in Figure la, the center of the circle has been offset vertically, by an amount defined by the angle $\mathrm{C}$ shown. The radius of the circle is enlarged from its undistorted value of $(1 / 2) \delta_{r}$ by factor $\mathrm{f}$, where

$$
f=\left(1+e^{2} \sigma^{2} / \delta^{2}\right)^{1 / 2}=\sec B .
$$

For 1-D structure, the circle is as shown in Figure 6a row (2): of radius $(1 / 2) \boldsymbol{e} \sigma$, and with the center on the Z'xy-axis. Angle $\mathrm{C}$ is zero, and the diagram has the characteristics of a 2-D case.

For the contrasting special case of high anisotropy, the circle takes the form shown in Figure 6a row (3). This circle is distinctive in going through the origin, (a result of the high anisotropy approximation which in effect sets $b$ to zero; in practice, such a circle would be close to the origin, but not through it).

For weak distortion, Figure 6a row (4), the values of $\sigma, d$, and $e$ can be read directly off the graph, with little approximation; and for strong distortion, characterized in Figure 6a row (5) with $|e| \rightarrow 1$, values of a and $S$ are again given graphically.

2) Twist but no shear $(t \neq 0$, e $=0)$; Figure 6 , column (b).-The general case for twist only is shown in Figure $6 b$ row (1). Again, relative to the undistorted tensor of Figure la the center of the circle has been offset vertically, by twist angle $A$. The radius of the circle has been enlarged by factor $\sec \mathrm{A}$.

For a twist with a 1-D case, Figure $6 \mathrm{~b}$ row (2), there is the unusual result of an offset single point, so that rotation of axes produces no effect. As is general for column $6 \mathrm{~b}$, the offset is by the twist angle $A$.

High anisotropy, Figure $6 \mathrm{~b}$ row (3), brings the circle through the origin; and weak distortion, Figure 6b row (4), produces a circle of simple description, from which values of $\boldsymbol{\sigma}, \mathrm{S}$, and $\boldsymbol{t}$ can be read.
3) Both shear and twist; Figure 6, column (c).-The most general case is presented in Figure $6 \mathrm{c}$ row (1). The radius of the circle is enlarged from $(1 / 2) \delta$ by a factor $f$ where

$$
f=\left(1+t^{2}\right)^{1 / 2}\left(1+e^{2} \sigma^{2} / \delta^{2}\right)^{1 / 2}=\sec A \sec B .
$$

The center shifts to coordinates

$$
\frac{1}{2} \sigma(1-t e \delta / \sigma), \quad \frac{1}{2}(t \sigma+e \delta)
$$

and so is offset from the horizontal axis by angle $(A+C)$.

The 1-D case in Figure 6c row (2) is of regular 3-D form. The high-anisotropy case, Figure 6c row (3), again goes through the origin, as does the strong or 2-D distortion case, Figure 6c row (5).

Comments on the principal impedances

With Groom and Bailey (1989), it is of interest to pursue a traditional 2-D search for recovery of the principal impedances, carried out by minimizing

$$
\left|Z^{\prime} x x\right|^{2}+\left|Z^{\prime} y y\right|^{2}
$$

as a function of coordinate rotation angle $\boldsymbol{\theta}^{\prime}$. Expanded, this procedure is the minimization of

$$
\left|Z^{\prime} x x_{r}\right|^{2}+\left|Z^{\prime} x x_{q}\right|^{2}+\left|Z^{\prime} y y_{r}\right|^{2}+\left|Z^{\prime} y y_{q}\right|^{2} \text {. }
$$

Considering only the first and third terms, and with reference to Figure 6c row (1), $\left|Z^{\prime} x x_{r}\right|$ is a minimum where the circle crosses the $Z^{\prime} x y_{r}$-axis. However, for a given position on the circle of $Z^{\prime} x x_{r}, Z^{\prime} y y$, has a magnitude given by the value of $Z^{\prime} x x_{r}$ that is diametrically opposite (see Figure 4). Hence the minimum value of $\left|Z^{\prime} x x_{r}\right|^{2}+\left|Z^{\prime} y y_{r}\right|^{2}$ will be given by the points of maximum and minimum $Z^{\prime} x y$, values. These particular values, from inspection of the figure, are

$$
\frac{1}{2} \sigma(1-t e \delta / \sigma)+\frac{1}{2} \delta \sec A \sec B
$$

and

$$
\frac{1}{2} \sigma(1-t e \delta / \sigma)-\frac{1}{2} \delta \sec A \sec B \text {. }
$$

Similar results hold for the minimization of $\left|Z^{\prime} x x_{q}\right|^{2}+$ $\left|Z^{\prime} y y_{q}\right|^{2}$.

The values of rotation angle $\theta^{\prime}$ at which the maximum and minimum values of $Z^{\prime} x y_{r}$ occur can be found by seeking maximum and minimum values of the $Z_{0}$ function of Lilley (1976), which in the notation of the present paper means seeking maximum and minimum values of a function

$$
Z_{0}\left(\theta^{\prime}\right)=\frac{1}{2} \alpha_{1} \cos 2 \theta^{\prime}-\frac{1}{2} \alpha_{3} \sin 26^{\prime} .
$$

Some algebra produces the result, using equations ( $3 \mathrm{~b})$ and (3d), that

$$
Z_{0}\left(\theta^{\prime}\right)=\frac{1}{2} \delta \sec A \sec B \cos \left(A+B+28-28^{\prime}\right)
$$

so that the maximum and minimum values of $Z_{0}\left(\theta^{\prime}\right)$ can be seen to occur when

$$
A+B+2 \theta-2 \theta^{\prime}=n \pi, \quad \text { where } n=0, \pm 1, \pm 2 \ldots .
$$
i.e., when

$$
\theta^{\prime}=\frac{1}{2}(A+B)+\theta-\frac{1}{2} n \pi .
$$

Thus when real and quadrature parts are considered separately, a general version of Groom and Bailey's (1989) result 
(their equation 41a) is obtained: that the directions found by minimizing and maximizing $Z^{\prime} x y$, will differ from the principal strike directions by $(1 / 2)(\mathrm{A}+\mathrm{B})$. Note that generally $(1 / 2)(A+B)$ is not half the skew angle, which is (112) $(A+C)$. In the high-anisotropy case considered by Groom and Bailey, for then $\mathrm{B}=\mathrm{C}$.

Also, of course, the maximum and minimum values found for $Z^{\prime} x y$, , as given above, are not the regional 2-D values of $[(1 / 2) \sigma+(1 / 2) \delta]$, and $[(1 / 2) \sigma-(1 / 2 \delta)]$; there appears to be no direct graphical method for identifying these latter (though they can be read off the graph in some particular cases such as those of weak distortion, given in Figure 6).

\section{Discussion}

The diagrams in Figure 6 make some particular points as follows.

1) A locally-sheared but regional 1-D case, as in Figure 6a row (2), is indistinguishable from a simple regional 2-D case, as in Figure la.

2) A locally-twisted but regional 1-D case, Figure 6b row (2), produces a distinctive simple point plot.

3) High regional 2-D anisotropy and strong local 2-D distortion both cause the circle to go near the origin, so there is one orientation of axes that gives low values for both Z'xx and Z'xy.

4) In view of the discussion concerning the invariants in Figure 4, Figure 6 shows that both high anisotropy and strong or 2-D distortion will have the effect of reducing the determinantal impedance (taking real parts of the tensor elements only) to near zero, while the second effective (or "trace") impedance is changed much less from its ideal value of (1/2)a. This point, for real and quadrature parts taken separately, may be relevant to the discussion in Groom and Bailey (1989) (and elsewhere) on the relative effects of distortion on the determinantal and trace impedances.

Generally, diagrams for the Groom-Bailey case of shear and twist have the form of the 3-D case of Figure lb, with the implication that a typical 3-D tensor may be subjected to Groom-Bailey analysis. The criterion for such analysis to be valid is that both the real and quadrature parts of a tensor should yield the same set of e, t, and $\theta$ values.

\section{CONCLUSIONS}

In their recent review, Wannamaker and Hohmann (1991) refer to a current revolution in electromagnetic induction studies. The magnetotelluric method has been central in this development, with data of increased precision observed over wide ranges of frequency. With modern computer software available for data reduction, there is still a need (indeed perhaps an increased need) for a display of diagnostic characteristics that help the judgements of an interpreter.

The Mohr circle method may be used to display magnetotelluric data succinctly, in the same way that it has proved useful for other tensor information. A visual impression is given of two-dimensionality, three-dimensionality, anisotropy, and skew, and insight is gained into the various magnetotelluric invariants and particular rotation angles.

\section{ACKNOWLEDGMENTS}

The author acknowledges beneficial discussions on magnetotelluric matters with Jean H. Filloux, Ian J. Ferguson, and Graham S. Heinson. Richard C. Bailey made helpful comments on an earlier version of the manuscript, and the reports of the referees have been of great value. Win Means is thanked for stimulating discussion of Mohr circles.

\section{REFERENCES}

Bahr, K., 1988, Interpretation of the magnetotelluric impedance tensor: Regional induction and local telluric distortion: J. Geophys., $62,119-127$.

- 1991, Geological noise in magnetotelluric data: A classification of distortion types: Phys. Earth Planet. Int., 66, 24-38.

Berdichevsky, M. N., and Dmitriev, V. I., 1976, Basic principles of interpretation of magnetotelluric curves, in Adam., Ed., Geoelectric and geothermal studies: Akademiai Kaido Budapest, KAPG Geophys. Mono., 165-221.

Counil, J. L., LeMouel, J. L., and Menvielle, M., 1986, Associate and conjugate directions concepts in magnetotellurics: Ann. Geophys., B4, 115-130.

Eggers, D. E., 1982, An eigenstate formulation of the magnetotelluric impedance tensor: Geophysics, 47, 12061214.

Ferguson, I. J., 1988, The Tasman project of seafloor magnetotelluric exploration: Ph.D. thesis, Australian National University,

Ferguson, I: J., Filloux, J. H., Lilley, F. E. M., Bindoff, N. L., and Mulheam, P. J., 1985, A seafloor magnetotelluric sounding in the Tasman Sea: Geophys. Res. Lett., 12, 545-548.

Ferguson, I. J., Lilley, F. E. M., and Filloux, J. H., 1990, Geomagnetic induction in the Tasman Sea and electrical conductivity structure beneath the Tasman seaflloor: Geophys. J. Int., 102, 299-312.

Filloux, J. H., 1987, Instrumentation and experimental methods for oceanic studies, in Jacobs, J. A., Ed., Geomagnetism: Academic Press, vol. 1, 143-247.

Groom, R. W., and Bailey, R. C., 1989, Decomposition of magnetotelluric impedance tensors in the presence of local threedimensional galvanic distortion: J. Geophys. Res., 94, 1913-1925.

- 1991, Analytic investigations of the effects of near-surface three-dimensional galvanic scatterers on MT tensor decompositions: Geophysics, 56, 496-518.

Heinson, G. S., 1991, Interpretation of seafloor magnetotelluric data using thin-sheet modeling: Ph.D. thesis, Australian National University, Canberra.

Ingham, M. R., 1988, The use of invariant impedances in magnetotelluric interpretation: Geophysical, J., 92, 165-169.

Jaeger, J. C., 1969, Elasticity, fracture and flow (3rd ed.): Methuen.

Jaeger, J. C., and Cook, N. G. W., 1976, Fundamentals of rock mechanics (2nd Ed.): John Wiley \& Sons, Inc.

LaTorraca, G. A., Madden, T. R., and Korringa, J., 1986, An analysis of the magnetotelluric impedance for three-dimensional conductivity structures: Geophysics, 51, 1819-1829.

Lilley, F. E. M., 1976, Diagrams for magnetotelluric data: Geophysics, $41,766-770$

Lilley, F. E. M., Filloux, J. H., Ferguson, I. J., Bindoff, N. L., and Mulheam, P. J., 1989, The Tasman project of seafloor magnetotelluric exploration: Experiment and observations: Phys. Earth Planet, Int., 53, 405-421.

Nye, J. F., 1957, Physical properties of crystals: Oxford Press.

Park, S. K., 1985, Distortions of magnetotelluric sounding curves by three-dimensional structures: Geophysics, 50, 785-797.

Park, S. K., and Livelybrooks, D. W., 1989, Quantitative interpretation of rotationally invariant parameters in magnetotellurics: Geophysics, 54, 1483-1490.

Spitz, S., 1985, The magnetotelluric impedance tensor properties with respect to rotations: Geophysics, 50, 1610-1617.

Swift, C. M., Jr., 1967, A magnetotelluric investigation of an electrical conductivity anomaly in the southwestern United States: Ph.D. thesis, Mass. Inst. of Tech.

Wannamaker, P. E., and Hohmann, G. W., 1991, Electromagnetic induction studies: Rev. Geophys. Supplement (U.S. Natl. Rep. to IUGG 1987-1990), 405-415.

Yee, E., and Paulson, K. V., 1987, The canonical decomposition and its relationship to other forms of magnetotelluric impedance tensor analysis: J. Geophys., 61, 173-189.

Zhang, P., Roberts, R. G., and Pedersen, L. B., 1987, Magnetotelluric strike rules: Geophysics, 51, 267-278. 